\title{
Bacillus subtilis Chassis in Biomanufacturing 4.0
}

\author{
Wenbo Zhang ${ }^{1}$, Xinshu Zhu ${ }^{2}$, Yan Wang ${ }^{1}$, and Tao $\mathrm{Li}^{1}$ \\ ${ }^{1}$ Xinxiang Medical University \\ ${ }^{2}$ Jiangsu College of Nursing
}

September 24, 2021

\begin{abstract}
Synthetic biology, an emerging research field, can promote biomanufacturing by offering various efficient chassis. Engineering Bacillus subtilis, an important workhorse in industrial biotechnology, through synthetic biology approaches may be a disruptive innovation. Advancements in chassis engineering, a synthetic biology strategy for genome-reduced cell factories, cell-free systems, and synthetic microbial consortia would be a driving force facilitating microbial production. We discussed chassis engineering categories and applications for B. subtilis. Prospects and challenges for chassis engineering in B. subtilis were also analyzed in this review article.
\end{abstract}

\section{Bacillus subtilis Chassis in Biomanufacturing $\mathbf{4 . 0}$}

Zhang Wenbo,11Correspondence to: zhangwenbo@xxmu.edu.cn; Zhang Wenbo (http://orcid.org/0000-00031661-3074), Xinxiang Medical University, Xinxiang, Henan 453003, China. +86-373-3831593 (Office); +86373-3029887 (Fax) $)^{\mathrm{a}}$ Zhu Xinshu ${ }^{\mathrm{b}, \mathrm{a}}$, Wang Yan ${ }^{\mathrm{a}}$, Li Tao ${ }^{\mathrm{a}}$

${ }^{a}$ Synthetic Biology Engineering Laboratory of Henan Province (SynBelHn), School of Life Sciences and Technology, Xinxiang Medical University, Xinxiang, Henan 453003, China

b College of Medical Technology, Jiangsu College of Nursing, No.9 Keji Road, Huai'an, Jiangsu 223001, China

Abstract : Synthetic biology, an emerging research field, can promote biomanufacturing by offering various efficient chassis. Engineering Bacillus subtilis, an important workhorse in industrial biotechnology, through synthetic biology approaches may be a disruptive innovation. Advancements in chassis engineering, a synthetic biology strategy for genome-reduced cell factories, cell-free systems, and synthetic microbial consortia would be a driving force facilitating microbial production. We discussed chassis engineering categories and applications for B. subtilis . Prospects and challenges for chassis engineering in B. subtilis were also analyzed in this review article.

Keywords : Bacillus subtilis ; Biomanufacturing; Cell-free system; Chassis; Genome reduction; Synthetic microbial consortium

\section{Introduction}

Biomanufacturing can crucially contribute to sustainable human development by utilizing biological systems to convert simple molecules, naturally occurring materials and even waste to value-added chemicals and biopolymers. Of note, Synthetic Biology (SynBio) offers disruptive technology for biomanufacturing, denoted as biomanufacturing 4.0 (BM 4.0) (Fig. 1) [1]. Recent developments in smart biomanufacturing and Biological Design Automation usher BM 4.0 into a new era (Table 1) [2-4].

The application of chassis in biomanufacturing 4.0 and the emergence of enabling technologies would drive the translation of the SynBio chassis from laboratory to industrial applications by minimizing regulating 
complexity and enhancing biological entity performance predictability, although synthetic biologists originally expected to study chassis, also referred to as the "living machine", as a way to decipher the nature of life [5].

Bacillus subtilis, a robust, fieldable and well-characterized host is likely one of the most promising chassis for biomanufacturing $4.0[6,7]$, considering that B. subtilis can biosynthesize value-added products such as single-chain antibodies, nucleotides, vitamins, antibiotics, biosurfactants, carbohydrates, and biodegradable polymers [8-13].

To offer an efficient, compatible and robust expression environment for aimed biosynthesis modules, B. subtilis , the host cell should be modified with SynBio approaches according to certain engineering principles, which can be termed as chassis engineering.

Some excellent reviews summarized synthetic biology toolbox and strategies for improving B. subtilis chassis performances $[7,14,15]$. However, they did not explain why different forms of chassis are essential for biomanufacturing 4.0. This mini-review will initially explain the definition, selection and categories of chassis. Then, we discussed the reasons why B. subtilis is suitable for biomanufacturing 4.0. Next, we highlighted progress in cell-free systems and synthetic microbial consortia, which are promising for biomanufacturing 4.0 as alternative forms of chassis. Finally, the challenges in the industrial applications of chassis and the next-generation chassis were analyzed, which may also be applicable for engineering chassis of other species like $B$. licheniformis for biomanufacturing [16].

\section{Biomanufacturing requires "better" chassis}

A chassis, a machine metaphor of a host cell, is an engineered life system that provides resources such as gene expression, protein translation and metabolic precursors, and it is also the carrier of biological components and devices, providing a predictable environment according to the principles in engineering practice, such as abstraction, modularity, orthogonality, robustness and compatibility to artificial components [17].

In a laboratory setting, chassis engineering refers to an engineering process that transforms existing natural biological systems or synthesizes artificial life systems from scratch, with the goal of providing an environment to enhance the robustness of biological parts (components) and devices (Table 1).

The basic strategy for current chassis engineering is to initially confirm the essentiality of each gene and then streamline the genome to build a "minimal cell". Successful construction of the minimal genomic JCVI-syn1.0 and JCVI-syn3.0 indicated the feasibility of the current research roadmap [18]. However, we speculate that chassis should not be limited to "minimal genomic cells", and chassis engineering should not be confined to "minimal genomic cells".

First, the definition of essentiality is context-dependent because it is related to cell culture conditions. Hence, the degree of genome reduction needs to be determined according to the defined living environments. Thus, the genome reduced cell or "minimal cell" is a relative concept. Second, the identification of "essential genes" can be influenced by research techniques. For example, not all essential genes belong to the paleome, the so-called persistent genes identified by sequence alignments reflecting bacterial original niches, causing inaccuracy in predicting essential genes by in silico methods [19, 20]. In addition, chassis in industrial environments and laboratory settings must meet different requirements. "Industrial chassis" require excellent robustness for adverse industrial environments. Therefore, chassis engineering for industry will be a process of installing artificial metabolic pathways or functional modules with the aid of enabling tools for designing, debugging and optimizing the expected functions of microbial manufacturing into genome-reduced chassis. In other words, industrial application of chassis engineering will be a process of not only genome reduction but also addition of certain functional modules. Accordingly, the degree of genome minimization needs to be tested and validated by wet experiments, not by computer prediction alone. Furthermore, the so-called minimal cells have not been widely exemplified in large-scale industrial applications; many challenges remain in the translation of genome-reduced chassis from laboratory to industry. Therefore, it is necessary to select acceptable chassis, appropriate forms and a right engineering roadmap, which would be explicitly explained 
as follows.

\section{More chassis options for biomanufacturing}

Selecting suitable platform cells or chassis is the initial step for biomanufacturing 4.0. The ideal platform cells that can be used for scale-up production should have the advantages of fast growth rate, high energy conversion efficiency, simple culture conditions, well-documented genetic background, desired tolerance to substrates and target products, as well as broad environmental adaptability [21]. Laboratory chassis, for example Escherichia coli and Saccharomyces cerevisiae, often fall short of these requirements to some extent, but $B$. subtilis is expected to be developed as an industrial chassis. Several advantages of B. subtilis chassis are summarized hereinafter from an industrial perspective. The reported minimal genomic chassis (MGC) is the current representative of single-celled chassis (Fig. 2). However, we speculate that chassis should not be limited to unicellular MGC. Other forms of chassis, such as cell-free systems (CFSs) and synthetic microbial consortia (SMC), may also have some basic characteristics of chassis.

\section{Bacillus subtilis: promising chassis}

To select and develop an industrial microbial workhorse as a cell factory, several factors should be considered, for instance, metabolic resources (e.g., precursors and cofactors), cell secretion capacity, product toxicity, genome manipulation toolset, cultural conditions, feasibility of large-scale cultivation, cost, and proper protein fold $[17,22,23]$. Suitable chassis can also be screened through bioinformatics tools following engineering principles such as compatibility between parts and chassis, availability of parts and cell growth rate [24, 25]. Comparing B. subtilis with other industrial workhorses, such as Pseudomonas putida ,Geobacillus sp., Deinoccocus sp., Corynebacterium glutamicum, Streptomyces sp., Clostridium acetobutylicum, Rhodococcus opacus and cyanobacteria, we speculate that $B$. subtilis has some unique advantages in exploiting synthetic biology techniques in biomanufacturing $[6,17,26]$.

B. subtilis is a Gram-positive sporiferous model microorganism with a long history of application in industry [7, 8, 27-29]. Some unique merits of B. subtilis make it appropriate for microbial manufacturing [30-32]. For example, several bulk industrial enzymes were produced by B. subtilis $[9,33]$. Engineered B. subtilis cells can also be used as biosensors, probiotics or vaccine delivery systems [8, 10, 34-36]. Additionally, $B$. subtilishas some benefits not found in laboratory chassis. Although B. subtilis has a simple cell envelope, it is highly resistant to omnipresent stress. This bacterium can assimilate cheap substrates, secrete a high titer of target proteins, induce competent or super-competent state, and accommodate large fragments of foreign DNA $[6,17,37]$. In contrast to $E$. coli , B. subtilis has no endotoxin and avoids the step of removing endotoxin in biopharmaceutical processes. Therefore, it is easier to produce extracellular products using $B$. subtilis than using $E$. coli or $S$. cerevisiae .

However, B. subtilis still has some disadvantages in contrast to laboratory chassis and some other industrial strains. For example, its product yield is not high enough and its growth cycle is relatively long. As to synthetic biological enabling tools, their development is chiefly based on E. coli and S. cerevisiae. Thus, it is crucial to develop novel enabling techniques for the $B$. subtilischassis $[6,38,39]$. By understanding the molecular mechanisms of $B$. subtilis related to productivity and robustness, as well as improving the existing enabling tools, we will be able to overcome its downsides and promote its application in biomanufacturing. Selecting appropriate chassis forms may be one way to mitigate the weakness of $B$. subtilis. We will discuss MGC first and then move on to CFS and SMC.

\section{Genome-reduced chassis}

The genome of B. subtilis has been sequenced and annotated, showing it is approximately $4.4 \mathrm{Mbp}$ in length and contains around 4100 genes [32, 40]. During evolution, B. subtilis continually absorbs foreign genetic resources by horizontal gene transfer [41]. Even though horizontal gene transfer may enhance bacterial adaptability to various niches, extra genes cause waste of energy and aggravate the complexity of product separation as well as purification.

Minimizing microbial genomes by deleting non-essential genes is expected to provide a predictable and 
phenotypically-controllable chassis. Utilizing global transposon mutation, comparative genomics, single gene knockout, clustered regularly interspaced short palindromic repeat interference (CRISPR interference or CRISPRi) and other techniques, it is predicted that B. subtilis has about 300-500 essential genes [42-44]. Other methods, such as machine learning, may also be applicable for gene essentiality identification for $B$. subtilis. SubtiWikilisted 251 essential genes encoding proteins and two essential RNA-coding genes for $B$. subtilis as of November 2020 [45]. These essential genes are mainly involved in protein synthesis and quality control, metabolism, cell envelope synthesis and cell division, DNA replication and modification, chromosome maintenance, RNA synthesis, RNA degradation as well as protective functions [30, 46].

A chassis with a reduced genome may have some advantages for biomanufacturing. For instance, genome reduction can improve the genetic stability of the strain by eliminating some horizontally transferred sequences, prophages and mobile genetic elements. After the genome is streamlined, the complexity of substances in culture media is reduced, which is expected to diminish the viscosity of the fermentation broth and suppress foaming by decreasing the production of biosurfactants (e.g., surfactin, iturin, and fengycin), thereby lessening the energy consumption of the fermentation process and facilitating oxygen delivery. Additionally, $B$. subtilis contains at least 20 proteases and peptidases. The stability of the expressed proteins can be improved after some protease genes are deleted. For instance, chassis such as MGB874 and PG10 can increase protein yield and produce proteins that are difficult to express [47, 48]. The non-essential genes currently deleted mainly include these categories: (1) genes related to genomic instability, such as $\operatorname{SP} \beta$ and PBSX prophage and pro1-7-type prophage; (2) regulatory genes; (3) stress-related genes; (4) large secondary metabolic operons, i.e., pks and pps ; and (5) sporulation genes, such as $\operatorname{kin} A, \operatorname{kin} B$, andkin $C$ [43, 49]. Genome reduction simplifies cell regulatory networks, similarly to "dimensionality reduction", an approach computer uses to deal with complex variables [50]. Moreover, genome-reduced cells may have higher electroporation efficiency and lower mutation rates [30]. In addition, genome streamlining will make the culture medium easier to design, improve bioprocess atomic economy, and facilitate product separation.

Even after the genome is simplified, the physiological characteristics of the chassis may not be improved. Therefore, further debugging and optimization is needed. Adaptive laboratory evolution (ALE) and genome editing technology might meet these expectations [51]. However, chassis usually remove DNA fragments that cause genomic instability. Accordingly, it may reduce evolvability. Hence, we speculate that the efficiency of ALE to improve chassis product performance, robustness and environmental pressure adaptability might decrease.

The efficiency of genome editing affects the efficiency of genome reduction. Thus, developing effective genome editing tools is significant for chassis construction. Some novel technologies exploiting bacterial defense systems offer appealing tools for genetic manipulation. For example, Wu et al . developed a novel counter-selectable cassette based on the B. subtilistoxin-antitoxin system to improve the efficiency of genome editing [52]. Compared to zinc-finger nucleases (ZFNs), transcription activator-like effector nucleases (TALENs) and some recombinase-based technologies, CRISPR-Cas9 is more efficient and will likely play an important role in B. subtilis chassis engineering [53-56]. CRISPR-related genome editing techniques may replace some traditional methods such as reverse selection, double-crossover marker-free genome editing, recombinase-based genome editing techniques and genome editing for strains that are difficult to transform [57-60]. Importantly, multiplex genome editing can greatly improve the efficiency of genome editing [61, 62]. Recently, CRISPR-Cas12a has emerged as a candidate for multiplex genome editing due to better specificity and more efficient crRNA array processing in comparison to CRISPR-Cas9 [63, 64]. Additionally, a retronbased genome editing system was successfully applied in the evolution of targeted sequences in $E$. coli , which suggests that retroelement-based editing tools may also work in B. subtilis [65].

\section{Cell-free chassis}

CFSs mainly refer to in vitro protein expression systems based on purified enzymes or cell extracts. Broadly speaking, cell-free artificial metabolism, cell-free transcription-translation system, systems biocatalysis, and ribosome display system can be generally classified as CFSs [66-68]. 
In addition to genome-reduced cells, CFSs can also be developed as chassis. For example, Kelwick et al . advanced a CFS based on B. subtilis WB800N [67]. This system did not use exogenous mRNAs, ribosomes, or protease inhibitors. Instead, it utilized a more efficient glycerol 3-phosphoglyceride exogenous energy buffer system to characterize a $B$. subtilis promoter library and expressed $0.8 \mu \mathrm{M}$ reporter protein GFPmut3b, which is a $\sim 2 \times$ fold-improvement when compared with its $B$. subtilis 168 counterparts. The lengthened reaction time was $150 \mathrm{~min}$ in contrast to previous reports $(15-60 \mathrm{~min})$. Tian et al . constructed a highly efficient B. subtilis cell-free system for N-acetylneuraminic acid (NeuAc) de novo synthesis, which had a 305-fold greater NeuAc synthesis rate than that in vivo and enabled fast pathway dynamics analysis [69]. They found that insufficient phosphoenolpyruvate supply was one of the NeuAc pathway's bottlenecks. Their study demonstrates that CFS provides a rapid method for metabolic pathway diagnosis (Fig. 3).

Developing B. subtilis CFS can be very rewarding if scientists take full advantage of the established CFSs. Presently, there are several well-established cell-free system preparation protocols and some commercialized systems. For example, PURE (Protein Synthesis Using Recombinant Elements), a reconstituted system, can be considered as a set of "standardized parts [70-72]."

CFSs have many benefits over cellular systems. Traditionally, chassis are intuitively assumed as living cells that provide metabolic and energy resources. However, cells have natural limitations. For example, cell growth requires specific temperatures and culture conditions, and certain toxic proteins or membrane proteins cannot be expressed. In fact, biological parts could be exploited to produce "minimized cells" using a bottom-up process [73]. Thus, CFSs provide a new option for producing proteins and accelerating the DBTL cycle in some biofoundries [66, 74]. Additionally, CFSs can function over a wide range of operating parameters (temperature, $\mathrm{pH}$, co-solvents, etc.), which can be homogeneous solution systems that are not limited by the kinetics of substrate diffusion or transmembrane active transport. Therefore, high yields are expected under the high load of substrates. In addition, CFSs are open systems that are easy to monitor, power, and modify by installing genetic circuits or metabolic pathways. Hence, CFSs offer an alternative to producing proteins that are difficult to express or toxic to the host, expanding the range of substrates.

CFSs are easy to prepare, preserve and measure. Hence, their application range is expanding to protein micro-fabrication, non-natural amino acid protein synthesis, protein glycosylation, non-ribosomal peptide synthesis, cyclic peptide synthesis, enzyme screening, prototyping of DNA regulation networks, biosensing, etc [68, 75-77]. CFSs are ideal for optimizing metabolic pathways and enabling artificial metabolic engineering to help produce substances that are incompatible with life systems [78]. If a CFS is constructed on the basis of minimized cells, its energy utilization may be more efficient. In addition, it is possible to further reduce genome size by optimizing the energy supply system and deleting scores of essential genes that are required for cell envelope, shape, and division [79].

Admittedly, CFSs have some drawbacks, for instance, high cost, short expression lifespan, and low efficiency in contrast to cellular systems due to requiring exogenous mRNAs, energy, and protein inhibitors. Additionally, the cell envelope of a CFS is removed, which may compromise compatibility between enzymes and modules. Consequently, biological reaction constraints should be re-examined in CFSs, such as the thermodynamic parameters and the optimum $\mathrm{pH}$ of enzymes. Fully exploiting thermostable enzymes developed through protein engineering may usher CFS into the new biomanufacturing age [80, 81].

\section{Synthetic microbial consortia}

SMC are artificial multi-cellular interaction systems that simulate the natural microbial community. Microbial communities often exhibit a population-level phenotype of complexity, relative stability, evolution and fluctuation. Traditionally, some fermentation products are produced by natural microbial communities, mainly mixed-species solid-state fermentation. Novel natural products can be discovered by studying the interactions between microorganisms [82]. In addition, designing artificial microbial communities can be useful for understanding and engineering microbiomes, that is, the whole microbiota in a human or animal body [83, 84]. Overall, SMC are possibly emerging as chassis for biomanufacturing (Fig. 4) [85-88].

B. subtilis -based SMC may be developed as chassis. For example, Hays et al . reported a stable (for weeks 
to months) and robust platform for producing amylase and polyhydroxybutyrate (PHB) by engineering autotrophic sucrose-secreting cyanobacteria (Synechococcus elongatus PCC 7942) and heterotrophic microorganisms (B. subtilis , E. coli, or S. cerevisiae ) [89]. These light-driven co-culture dyads revealed general design principles for the construction of robust autotroph/heterotroph consortia as photoproduction platforms, which can persist when challenged with specific perturbations. Additionally, it was reported that bioplastic producing Ralstonia eutropha 5119 and sucrose hydrolyzing B. subtilis were co-cultured as SMC that can manufacture poly(3-hydroxybutyrate-co-3-hydroxyvalerate) copolymer from sugarcane without precursor feeding [90]. Liu et al . built a consortium as a microbial fuel cell consisting of B. subtilis RH33 and Shewanella oneidensis MR-1, which generates bioenergy stably for $500 \mathrm{~h}$ [91]. Electron transfer efficiency of this consortium was enhanced because MR-1 could efficiently utilize the high concentration of riboflavin produced by RH33. These instances show that SMC may have advantages over MGC in terms of energy/carbon source utilization.

Apart from the above-mentioned multispecies system, there are some seminal reports discussing monospecies SMC, which are promising for microbial production. Hong et al . designed a genetic circuit based on the quorum sensing (QS) system, which can be used to achieve competition between strains, and replace the biofilm of another strain [92]. One advantage of a monospecies SMC is that there is no need to optimize the medium.

Research shows that SMC have many unique advantages over monoculture (pure culture) systems because they can utilize complex substrates, reduce the metabolic burden of hosts and lessen genetic burden by avoiding assembling too many genetic circuits [93, 94]. Currently, different types of chassis are applied toward biomanufacturing and enabled by integrating functional modules or rewiring synthetic gene regulatory networks. As functional modules and synthetic gene regulatory network complexity grows, it is difficult to coordinate these modules and networks within a pure culture system. Using SMC is advantageous for assembling different biological circuits, functional modules or metabolic pathways to different cells and avoiding incompatibility among synthetic biological devices/modules/pathways [95]. Hence, SMC compartmentalizes various functional modules so that different strains can perform distinct functions simultaneously with reduced interference in order to implement complex functions [96]. Meanwhile, new products can be synthesized by exploiting enzyme resources from diverse strains. SMC assist scientists to understand multi-cellular temporal and spatial dynamic behavior and population stability [88]. Additionally, SMC may be more resistant to environmental factors and able to respond to changing environments [92]. Moreover, SMC can increase the yield of biomass, which is desirable for biomanufacturing [97].

Introducing artificial cell-to-cell communication and metabolite exchange are the main principles for designing synthetic microbial communities [98, 99]. Cell-signaling mechanisms (such as QS) can be applied to create synthetic circuits across cells from the bottom up that enable cells to exhibit population behavior [100, 101]. Artificial microbial communities can also be reconstructed from the top down on the basis of natural microbial communities. Furthermore, systems biology offers insights into the rational design of synthetic microbial ecosystems [83].

By engineering B. subtilis' signal transduction and metabolite exchange, scientific workers can rationally engineer B. subtilisfor applications in medicine, food and feed sectors [10,11]. Although there are various interactions among cells, positive interactions (e.g., commensalism, mutualism) are desirable for biomanufacturing. Thus, it is valuable to investigate mechanisms for positive interactions as well as those factors impacting microbial ecosystems' genetic stability and long-term robustness. Additionally, biofilm-related molecular mechanisms and the multicellular nature of B.subtilis might be useful for designing monospecies SMC [102]. Furthermore, most reported SMC are composed of wild-type strains or engineered strains. If SMC were built from MGC, its physiological behavior could be more predictable than current counterparts.

Undoubtedly, current B. subtilis -based SMC also have some imperfections, for instance, few applications and difficulties in wiring logic computing [103-105]. Additionally, few B. subtilis -based SMC have been developed due to insufficient understanding of intercellular communications and interspecies metabolite utilization. However, we believe that SMC will offer new options as chassis for biomanufacturing and environmental 
remediations through applying new approaches such as QS engineering and microfluidic chips $[92,106]$.

\section{Concluding remarks}

This review article suggests that B. subtilis chassis may have three categories: MGC, CFS and SMC. They have great challenges and potential in biomanufacturing 4.0. For example, genome-reduced B. subtilis chassis has demonstrated superior production characteristics to some extent. Moreover, with the development of highly efficient DNA synthesis, it is possible to synthesize the B. subtilis genome from the bottom up in the future [18]. In addition to MGC, other types of chassis can be expected to expand their application scenarios. For example, synthesizing some high-value proteins in CFS seems commercially feasible, although it is still in its infancy. In addition, well-developed B. subtilis -based SMC can be achieved by engineering QS systems, reconstructing artificial-metabolic circuits and exploiting metabolic cross-feeding in the forthcoming days [107].

To fully exploit B. subtilis chassis' potential in biomanufacturing in the future, we should focus on essentialome (this term is coined herein to stand for a total set of essential genes ) engineering.

Currently, chassis engineering projects are chiefly aiming at exploring the way to streamline non-essential genes to increase "atomic economy" for biomanufacturing and to decrease the biological regulating network complexity to strengthen predictability and genetic stability. Existing essential genes usually belong to the paleome, which determines cell doubling [20]. The paleome codes translation and transcription machines and provides information services for biosynthesis. Ribosomes, the core device of translation machines, determine the efficiency of protein production $[108,109]$. Notably, engineering ribosomes can increase translation efficiency and temperature range $[110,111]$.

Accordingly, these examples suggest that the next generation of chassis engineering should focus on the essentialome, which codes transcription and translation machines and other necessary components for life persistence. We speculate that essentialome engineering may be an effective approach to breaking through the limitations of existing chassis because constraints within chassis can be fundamentally changed. Through essentialome engineering, next-generation chassis will not contain the original set of essential genes; instead, they will be composed of novel transcription and translation machines developed by evolution engineering, genetic code expansion, genome recoding, synthetic chimeric genome or other techniques [110-112]. Nextgeneration chassis derived from essentialome engineering may have not only novel ribosomes to decode metabolic pathway information with high efficiency but also sharper senses to interpret environmental signals.

Taken together, there is a growing demand to develop B. subtilis -based chassis for biomanufacturing 4.0 [113]. These challenges in chassis engineering can be solved by the synthetic biologist community and enabling technology democratization.

\section{Funding:}

This article was supported by the Doctoral Scientific Research Grant of Xinxiang Medical University (No. 100824).

\section{Conflicts of interest}

All authors report no conflict of interest.

\section{References}

[1] Zhang, Y. P., Sun, J., Ma, Y., Biomanufacturing: history and perspective. J Ind Microbiol Biotechnol 2017, $44,773-784$.

[2] Carbonell, P., Le Feuvre, R., Takano, E., Scrutton, N. S., In silico design and automated learning to boost next-generation smart biomanufacturing. Synth Biol (Oxf) 2020, 5 , ysaa020.

[3] Gargalo, C. L., Udugama, I., Pontius, K., Lopez, P. C., et al. , Towards smart biomanufacturing: a perspective on recent developments in industrial measurement and monitoring technologies for bio-based 
production processes. J Ind Microbiol Biotechnol 2020,47, 947-964.

[4] Liu, Y., Su, A., Li, J., Ledesma-Amaro, R., et al. , Towards next-generation model microorganism chassis for biomanufacturing. Appl Microbiol Biotechnol 2020, 104, 9095-9108.

[5] Chi, H., Wang, X., Shao, Y., Qin, Y., et al. , Engineering and modification of microbial chassis for systems and synthetic biology.Synth Syst Biotechnol 2019, 4 , 25-33.

[6] Adams, B. L., The Next Generation of Synthetic Biology Chassis: Moving Synthetic Biology from the Laboratory to the Field. ACS Synth Biol 2016, 5 , 1328-1330.

[7] Gu, Y., Xu, X., Wu, Y., Niu, T., et al. , Advances and prospects of Bacillus subtilis cellular factories: From rational design to industrial applications. Metab Eng 2018, 50 , 109-121.

[8] Cui, W., Han, L., Suo, F., Liu, Z., et al. , Exploitation of Bacillus subtilis as a robust workhorse for production of heterologous proteins and beyond. World J Microbiol Biotechnol2018, 34 , 145.

[9] Jujjavarapu, S. E., Dhagat, S., Evolutionary Trends in Industrial Production of alpha-amylase. Recent Pat Biotechnol2019, 13 , 4-18.

[10] Ayala, F. R., Bauman, C., Cogliati, S., Lenini, C., et al. , Microbial flora, probiotics, Bacillus subtilis and the search for a long and healthy human longevity. Microb Cell 2017, 4, 133-136.

[11] Kumar, P., Patel, S. K., Lee, J. K., Kalia, V. C., Extending the limits of Bacillus for novel biotechnological applications.Biotechnol Adv 2013, 31 , 1543-1561.

[12] Kaspar, F., Neubauer, P., Gimpel, M., Bioactive Secondary Metabolites from Bacillus subtilis: A Comprehensive Review. J Nat Prod 2019, 82 , 2038-2053.

[13] Ma, W., Liu, Y., Lv, X., Li, J., et al. , Combinatorial pathway enzyme engineering and host engineering overcomes pyruvate overflow and enhances overproduction of N-acetylglucosamine in Bacillus subtilis. Microb Cell Fact 2019, 18 , 1.

[14] Liu, Y., Liu, L., Li, J., Du, G., Chen, J., Synthetic Biology Toolbox and Chassis Development in Bacillus subtilis. Trends Biotechnol 2019, 37, 548-562.

[15] Xiang, M., Kang, Q., Zhang, D., Advances on systems metabolic engineering of Bacillus subtilis as a chassis cell. Synth Syst Biotechnol 2020, 5 , 245-251.

[16] Li, T., Wang, H., Li, J., Jiang, L., et al. , Enzymatic characterization, molecular dynamics simulation, and application of a novel Bacillus licheniformis laccase. Int J Biol Macromol 2021,167, 1393-1405.

[17] Calero, P., Nikel, P. I., Chasing bacterial chassis for metabolic engineering: a perspective review from classical to non-traditional microorganisms. Microb Biotechnol 2019,12 , 98-124.

[18] Hutchison, C. A., 3rd, Chuang, R. Y., Noskov, V. N., Assad-Garcia, N., et al. , Design and synthesis of a minimal bacterial genome. Science 2016, 351 , aad6253.

[19] Acevedo-Rocha, C. G., Fang, G., Schmidt, M., Ussery, D. W., Danchin, A., From essential to persistent genes: a functional approach to constructing synthetic life. Trends in genetics : TIG 2013,29, 273-279.

[20] Danchin, A., Scaling up synthetic biology: Do not forget the chassis. FEBS Lett 2012, 586 , 2129-2137.

[21] Foley, P. L., Shuler, M. L., Considerations for the design and construction of a synthetic platform cell for biotechnological applications. Biotechnol Bioeng 2010, 105 , 26-36.

[22] Garcia-Ruiz, E., HamediRad, M., Zhao, H., Pathway Design, Engineering, and Optimization. Adv Biochem Eng Biotechnol 2018,162 , 77-116.

[23] Zhang, K., Su, L., Wu, J., Recent Advances in Recombinant Protein Production by Bacillus subtilis. Annu Rev Food Sci Technol 2020, 11 , 295-318. 
[24] Carbonell, P., Currin, A., Jervis, A. J., Rattray, N. J., et al. , Bioinformatics for the synthetic biology of natural products: integrating across the Design-Build-Test cycle. Nat Prod Rep2016, 33 , 925-932.

[25] Kim, B., Kim, W. J., Kim, D. I., Lee, S. Y., Applications of genome-scale metabolic network model in metabolic engineering. J Ind Microbiol Biotechnol 2015, 42 , 339-348.

[26] Nikel, P. I., Martinez-Garcia, E., de Lorenzo, V., Biotechnological domestication of pseudomonads using synthetic biology. Nat Rev Microbiol 2014, 12 , 368-379.

[27] Yang, S., Du, G., Chen, J., Kang, Z., Characterization and application of endogenous phase-dependent promoters in Bacillus subtilis. Appl Microbiol Biotechnol 2017, 101 , 4151-4161.

[28] Harwood, C. R., Pohl, S., Smith, W., Wipat, A., Chapter 4 - Bacillus subtilis: Model Gram-Positive Synthetic Biology Chassis, in: Harwood, C., Wipat, A. (Eds.), Methods in Microbiology , Academic Press 2013, pp. 87-117.

[29] Liu, Y., Liu, L., Li, J., Du, G., Chen, J., Synthetic Biology Toolbox and Chassis Development in Bacillus subtilis. Trends Biotechnol 2018.

[30] Juhas, M., Reuss, D. R., Zhu, B., Commichau, F. M., Bacillus subtilis and Escherichia coli essential genes and minimal cell factories after one decade of genome engineering. Microbiology 2014,160 , 2341-2351.

[31] Commichau, F. M., Pietack, N., Stulke, J., Essential genes in Bacillus subtilis: a re-evaluation after ten years. Mol Biosyst2013, 9 , 1068-1075.

[32] Kunst, F., Ogasawara, N., Moszer, I., Albertini, A. M., et al. , The complete genome sequence of the gram-positive bacterium Bacillus subtilis. Nature 1997, 390 , 249-256.

[33] Liu, Y., Li, J., Du, G., Chen, J., Liu, L., Metabolic engineering of Bacillus subtilis fueled by systems biology: Recent advances and future directions. Biotechnol Adv 2017, 35 , 20-30.

[34] Kobras, C. M., Mascher, T., Gebhard, S., Application of a Bacillus subtilis Whole-Cell Biosensor (PliaIlux) for the Identification of Cell Wall Active Antibacterial Compounds.Methods Mol Biol 2017, 1520 , 121-131.

[35] Fantino, J. R., Barras, F., Denizot, F., Sposensor: a whole-bacterial biosensor that uses immobilized Bacillus subtilis spores and a one-step incubation/detection process. J Mol Microbiol Biotechnol 2009, 17, 90-95.

[36] Siedler, S., Khatri, N. K., Zsohar, A., Kjaerbolling, I., et al. , Development of a Bacterial Biosensor for Rapid Screening of Yeast p-Coumaric Acid Production. ACS Synth Biol 2017, 6 , 1860-1869.

[37] Inaoka, T., Kimura, K., Morimatsu, K., Yamamoto, K., Characterization of high hydrostatic pressureinjured Bacillus subtilis cells. Bioscience, biotechnology, and biochemistry 2017,81 , 1235-1240.

[38] Ohtani, N., Hasegawa, M., Sato, M., Tomita, M., et al. , Serial assembly of Thermus megaplasmid DNA in the genome of Bacillus subtilis 168: a BAC-based domino method applied to DNA with a high GC content. Biotechnol J 2012, 7 , 867-876.

[39] Appleton, E., Madsen, C., Roehner, N., Densmore, D., Design Automation in Synthetic Biology. Cold Spring Harb Perspect Biol2017, 9 .

[40] Moszer, I., The complete genome of Bacillus subtilis: from sequence annotation to data management and analysis. FEBS Lett1998, $430,28-36$.

[41] Tosato, V., Bruschi, C. V., Knowledge of the Bacillus subtilis genome: impacts on fundamental science and biotechnology. Appl Microbiol Biotechnol 2004, 64, 1-6.

[42] Glass, J. I., Assad-Garcia, N., Alperovich, N., Yooseph, S., et al. , Essential genes of a minimal bacterium. Proc Natl Acad Sci U S A 2006, 103 , 425-430. 
[43] Ara, K., Ozaki, K., Nakamura, K., Yamane, K., et al. , Bacillus minimum genome factory: effective utilization of microbial genome information. Biotechnol Appl Biochem 2007, 46 , 169-178.

[44] Peters, J. M., Colavin, A., Shi, H., Czarny, T. L., et al. , A Comprehensive, CRISPR-based Functional Analysis of Essential Genes in Bacteria. Cell 2016, 165 , 1493-1506.

[45] Michna, R. H., Commichau, F. M., Todter, D., Zschiedrich, C. P., Stulke, J., SubtiWiki-a database for the model organism Bacillus subtilis that links pathway, interaction and expression information.Nucleic Acids Res 2014, 42 , D692-698.

[46] Reuss, D. R., Commichau, F. M., Gundlach, J., Zhu, B., Stulke, J., The Blueprint of a Minimal Cell: MiniBacillus. Microbiol Mol Biol Rev 2016, 80 , 955-987.

[47] Morimoto, T., Kadoya, R., Endo, K., Tohata, M., et al. , Enhanced recombinant protein productivity by genome reduction in Bacillus subtilis. DNA Res 2008, 15 , 73-81.

[48] Aguilar Suarez, R., Stulke, J., van Dijl, J. M., Less is more: towards a genome-reduced Bacillus cell factory for 'difficult proteins'.ACS Synth Biol 2018.

[49] Westers, H., Dorenbos, R., van Dijl, J. M., Kabel, J., et al. , Genome engineering reveals large dispensable regions in Bacillus subtilis. Mol Biol Evol 2003, 20 , 2076-2090.

[50] Reuss, D. R., Altenbuchner, J., Mader, U., Rath, H., et al. , Large-scale reduction of the Bacillus subtilis genome: consequences for the transcriptional network, resource allocation, and metabolism. Genome Res 2017, 27, 289-299.

[51] Dragosits, M., Mattanovich, D., Adaptive laboratory evolution - principles and applications for biotechnology. Microb Cell Fact 2013, 12, 64.

[52] Wu, J., Deng, A., Sun, Q., Bai, H., et al. , Bacterial Genome Editing via a Designed Toxin-Antitoxin Cassette. ACS Synth Biol 2018, 7, 822-831.

[53] Schilling, T., Dietrich, S., Hoppert, M., Hertel, R., A CRISPR-Cas9-Based Toolkit for Fast and Precise In Vivo Genetic Engineering of Bacillus subtilis Phages. Viruses 2018, 10 .

[54] Westbrook, A. W., Ren, X., Oh, J., Moo-Young, M., Chou, C. P., Metabolic engineering to enhance heterologous production of hyaluronic acid in Bacillus subtilis. Metab Eng 2018, 47, 401-413.

[55] Price, M. A., Cruz, R., Bryson, J., Escalettes, F., Rosser, S. J., Expanding and understanding the CRISPR toolbox for Bacillus subtilis with MAD7 and dMAD7. Biotechnol Bioeng 2020, 117, 1805-1816.

[56] Yu, S., Price, M. A., Wang, Y., Liu, Y., et al. , CRISPR-dCas9 Mediated Cytosine Deaminase Base Editing in Bacillus subtilis. ACS Synth Biol 2020, 9 , 1781-1789.

[57] Zhang, K., Duan, X., Wu, J., Multigene disruption in undomesticated Bacillus subtilis ATCC 6051a using the CRISPR/Cas9 system. Sci Rep 2016, 6 , 27943.

[58] Wang, Y., Weng, J., Waseem, R., Yin, X., et al. , Bacillus subtilis genome editing using ssDNA with short homology regions. Nucleic Acids Res 2012, 40 , e91.

[59] Yan, X., Yu, H. J., Hong, Q., Li, S. P., Cre/lox system and PCR-based genome engineering in Bacillus subtilis. Appl Environ Microbiol 2008, 74, 5556-5562.

[60] Dong, H., Zhang, D., Current development in genetic engineering strategies of Bacillus species. Microb Cell Fact 2014, 13 , 63.

[61] Esvelt, K. M., Wang, H. H., Genome-scale engineering for systems and synthetic biology. Mol Syst Biol $2013,9,641$.

[62] Liu, D., Huang, C., Guo, J., Zhang, P., et al. , Development and characterization of a CRISPR/Cas9nbased multiplex genome editing system for Bacillus subtilis. Biotechnol Biofuels2019, 12 , 197. 
[63] Adiego-Perez, B., Randazzo, P., Daran, J. M., Verwaal, R., et al. , Multiplex genome editing of microorganisms using CRISPR-Cas. FEMS Microbiol Lett 2019, 366 .

[64] Wu, Y., Liu, Y., Lv, X., Li, J., et al. , CAMERS-B: CRISPR/Cpf1 assisted multiple-genes editing and regulation system for Bacillus subtilis. Biotechnol Bioeng 2020, 117, 1817-1825.

[65] Simon, A. J., Morrow, B. R., Ellington, A. D., Retroelement-Based Genome Editing and Evolution. ACS Synth Biol2018, 7 , 2600-2611.

[66] Fessner, W. D., Systems Biocatalysis: Development and engineering of cell-free "artificial metabolisms" for preparative multi-enzymatic synthesis. N Biotechnol 2015, 32 , 658-664.

[67] Kelwick, R., Webb, A. J., MacDonald, J. T., Freemont, P. S., Development of a Bacillus subtilis cell-free transcription-translation system for prototyping regulatory elements. Metab Eng 2016,38 , 370-381.

[68] Petroll, K., Kopp, D., Care, A., Bergquist, P. L., Sunna, A., Tools and strategies for constructing cell-free enzyme pathways.Biotechnol Adv 2019, 37 , 91-108.

[69] Tian, R., Wang, M., Shi, J., Qin, X., et al. , Cell-free synthesis system-assisted pathway bottleneck diagnosis and engineering in Bacillus subtilis. Synth Syst Biotechnol 2020, 5 , 131-136.

[70] Matsubayashi, H., Ueda, T., Purified cell-free systems as standard parts for synthetic biology. Curr Opin Chem Biol 2014,22, 158-162.

[71] Perez, J. G., Stark, J. C., Jewett, M. C., Cell-Free Synthetic Biology: Engineering Beyond the Cell. Cold Spring Harb Perspect Biol 2016, 8 .

[72] Shimizu, Y., Inoue, A., Tomari, Y., Suzuki, T., et al. , Cell-free translation reconstituted with purified components. Nat Biotechnol 2001, 19 , 751-755.

[73] Jia, H., Heymann, M., Bernhard, F., Schwille, P., Kai, L., Cell-free protein synthesis in micro compartments: building a minimal cell from biobricks. N Biotechnol 2017, 39 , 199-205.

[74] Karim, A. S., Jewett, M. C., Cell-Free Synthetic Biology for Pathway Prototyping. Methods Enzymol 2018, $608,31-57$.

[75] Swank, Z., Laohakunakorn, N., Maerkl, S. J., Cell-free gene-regulatory network engineering with synthetic transcription factors. Proc Natl Acad Sci U S A 2019, 116 , 5892-5901.

[76] Goering, A. W., Li, J., McClure, R. A., Thomson, R. J., et al. , In Vitro Reconstruction of Nonribosomal Peptide Biosynthesis Directly from DNA Using Cell-Free Protein Synthesis. ACS Synth Biol 2017, 6 , 39-44.

[77] Jaroentomeechai, T., Taw, M. N., Li, M., Aquino, A., et al., Cell-Free Synthetic Glycobiology: Designing and Engineering Glycomolecules Outside of Living Cells. Front Chem 2020,8 , 645.

[78] Jung, G. Y., Stephanopoulos, G., A functional protein chip for pathway optimization and in vitro metabolic engineering. Science2004, 304, 428-431.

[79] Kobayashi, K., Ehrlich, S. D., Albertini, A., Amati, G., et al. , Essential Bacillus subtilis genes. Proc Natl Acad Sci U S A 2003, 100 , 4678-4683.

[80] Ma, F., Xie, Y., Luo, M., Wang, S., et al. , Sequence homolog-based molecular engineering for shifting the enzymatic pH optimum. Synth Syst Biotechnol 2016, 1 , 195-206.

[81] Shi, T., Han, P., You, C., Zhang, Y. P. J., An in vitro synthetic biology platform for emerging industrial biomanufacturing: Bottom-up pathway design. Synth Syst Biotechnol 2018, 3 , 186-195.

[82] Derewacz, D. K., Covington, B. C., McLean, J. A., Bachmann, B. O., Mapping Microbial Response Metabolomes for Induced Natural Product Discovery. ACS Chem Biol 2015, 10 , 1998-2006. 
[83] Song, H., Ding, M. Z., Jia, X. Q., Ma, Q., Yuan, Y. J., Synthetic microbial consortia: from systematic analysis to construction and applications. Chem Soc Rev 2014, 43 , 6954-6981.

[84] Woloszynek, S., Pastor, S., Mell, J. C., Nandi, N., et al. , Engineering Human Microbiota: Influencing Cellular and Community Dynamics for Therapeutic Applications. Int Rev Cell Mol Biol2016, 324, 67-124.

[85] Pandhal, J., Noirel, J., Synthetic microbial ecosystems for biotechnology. Biotechnol Lett 2014, 36 , $1141-1151$.

[86] Tsoi, R., Dai, Z., You, L., Emerging strategies for engineering microbial communities. Biotechnol Adv 2019, 37, 107372 .

[87] Noack, S., Baumgart, M., Communities of Niche-Optimized Strains: Small-Genome Organism Consortia in Bioproduction. Trends Biotechnol 2019, 37, 126-139.

[88] Du, J., Jia, X., Yuan, Y., Research progress of synthetic microbial consortia. Journal of Chemical Industry and Engineering(China) 2014, 65 , 390-395.

[89] Hays, S. G., Yan, L. L. W., Silver, P. A., Ducat, D. C., Synthetic photosynthetic consortia define interactions leading to robustness and photoproduction. J Biol Eng 2017, 11 , 4.

[90] Bhatia, S. K., Yoon, J. J., Kim, H. J., Hong, J. W., et al. , Engineering of artificial microbial consortia of Ralstonia eutropha and Bacillus subtilis for poly(3-hydroxybutyrate-co-3-hydroxyvalerate) copolymer production from sugarcane sugar without precursor feeding. Bioresour Technol2018, 257, 92-101.

[91] Liu, T., Yu, Y. Y., Chen, T., Chen, W. N., A synthetic microbial consortium of Shewanella and Bacillus for enhanced generation of bioelectricity. Biotechnol Bioeng 2017, 114, 526-532.

[92] Hong, S. H., Hegde, M., Kim, J., Wang, X., et al. , Synthetic quorum-sensing circuit to control consortial biofilm formation and dispersal in a microfluidic device. Nat Commun 2012,3 , 613.

[93] Shong, J., Jimenez Diaz, M. R., Collins, C. H., Towards synthetic microbial consortia for bioprocessing. Curr Opin Biotechnol 2012, 23 , 798-802.

[94] Carbonell-Ballestero, M., Garcia-Ramallo, E., Montanez, R., Rodriguez-Caso, C., Macia, J., Dealing with the genetic load in bacterial synthetic biology circuits: convergences with the Ohm's law.Nucleic Acids Res 2016, 44 , 496-507.

[95] Macia, J., Manzoni, R., Conde, N., Urrios, A., et al. , Implementation of Complex Biological Logic Circuits Using Spatially Distributed Multicellular Consortia. PLoS computational biology2016, 12, e1004685.

[96] Fiore, G., Matyjaszkiewicz, A., Annunziata, F., Grierson, C., et al. , In-Silico Analysis and Implementation of a Multicellular Feedback Control Strategy in a Synthetic Bacterial Consortium. ACS Synth Biol 2017, $6,507-517$.

[97] Bernstein, H. C., Paulson, S. D., Carlson, R. P., Synthetic Escherichia coli consortia engineered for syntrophy demonstrate enhanced biomass productivity. J Biotechnol 2012, 157 , 159-166.

[98] Che, S., Men, Y., Synthetic microbial consortia for biosynthesis and biodegradation: promises and challenges. J Ind Microbiol Biotechnol 2019, 46 , 1343-1358.

[99] Jagmann, N., Philipp, B., Design of synthetic microbial communities for biotechnological production processes. J Biotechnol 2014, 184, 209-218.

[100] Chen, Y., Kim, J. K., Hirning, A. J., Josic, K., Bennett, M. R., SYNTHETIC BIOLOGY. Emergent genetic oscillations in a synthetic microbial consortium. Science 2015, 349 , 986-989.

[101] Scott, S. R., Hasty, J., Quorum Sensing Communication Modules for Microbial Consortia. ACS Synth Biol 2016, 5 , 969-977. 
[102] Aguilar, C., Vlamakis, H., Losick, R., Kolter, R., Thinking about Bacillus subtilis as a multicellular organism. Curr Opin Microbiol 2007, 10 , 638-643.

[103] Seccareccia, I., Kovacs, A. T., Gallegos-Monterrosa, R., Nett, M., Unraveling the predator-prey relationship of Cupriavidus necator and Bacillus subtilis. Microbiol Res 2016, 192 , 231-238.

[104] Ji, W., Shi, H., Zhang, H., Sun, R., et al. , A formalized design process for bacterial consortia that perform logic computing. PLoS One 2013, 8 , e 57482.

[105] Waite, A. J., Shou, W., Constructing synthetic microbial communities to explore the ecology and evolution of symbiosis. Methods Mol Biol 2014, 1151 , 27-38.

[106] Szita, N., Polizzi, K., Jaccard, N., Baganz, F., Microfluidic approaches for systems and synthetic biology. Curr Opin Biotechnol 2010, 21 , 517-523.

[107] Bulter, T., Lee, S. G., Wong, W. W., Fung, E., et al. , Design of artificial cell-cell communication using gene and metabolic networks. Proc Natl Acad Sci U S A 2004, 101 , 2299-2304.

[108] Gorochowski, T. E., Avcilar-Kucukgoze, I., Bovenberg, R. A., Roubos, J. A., Ignatova, Z., A Minimal Model of Ribosome Allocation Dynamics Captures Trade-offs in Expression between Endogenous and Synthetic Genes. ACS Synth Biol 2016, 5 , 710-720.

[109] Danchin, A., Fang, G., Noria, S., The extant core bacterial proteome is an archive of the origin of life. Proteomics 2007,7 , 875-889.

[110] Acevedo-Rocha, C. G., Budisa, N., Xenomicrobiology: a roadmap for genetic code engineering. Microb Biotechnol 2016, 9 , 666-676.

[111] Tizei, P. A., Csibra, E., Torres, L., Pinheiro, V. B., Selection platforms for directed evolution in synthetic biology.Biochemical Society transactions 2016, 44 , 1165-1175.

[112] Davidson, E. A., Windram, O. P., Bayer, T. S., Building synthetic systems to learn nature's design principles. Adv Exp Med Biol 2012, 751 , 411-429.

[113] Zhang W (2019) Marine Enzyme. In: C. Luo (ed) Marine Food and Technology . Science Press, Beijing, pp 227-243.

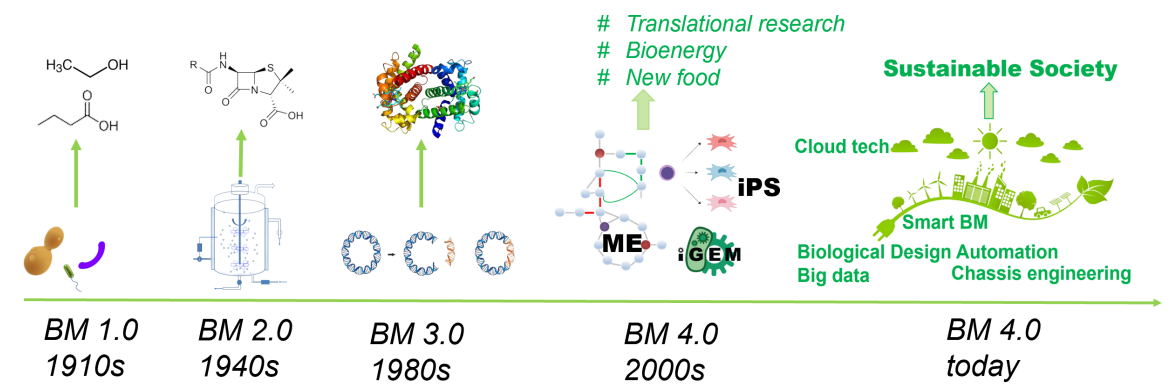



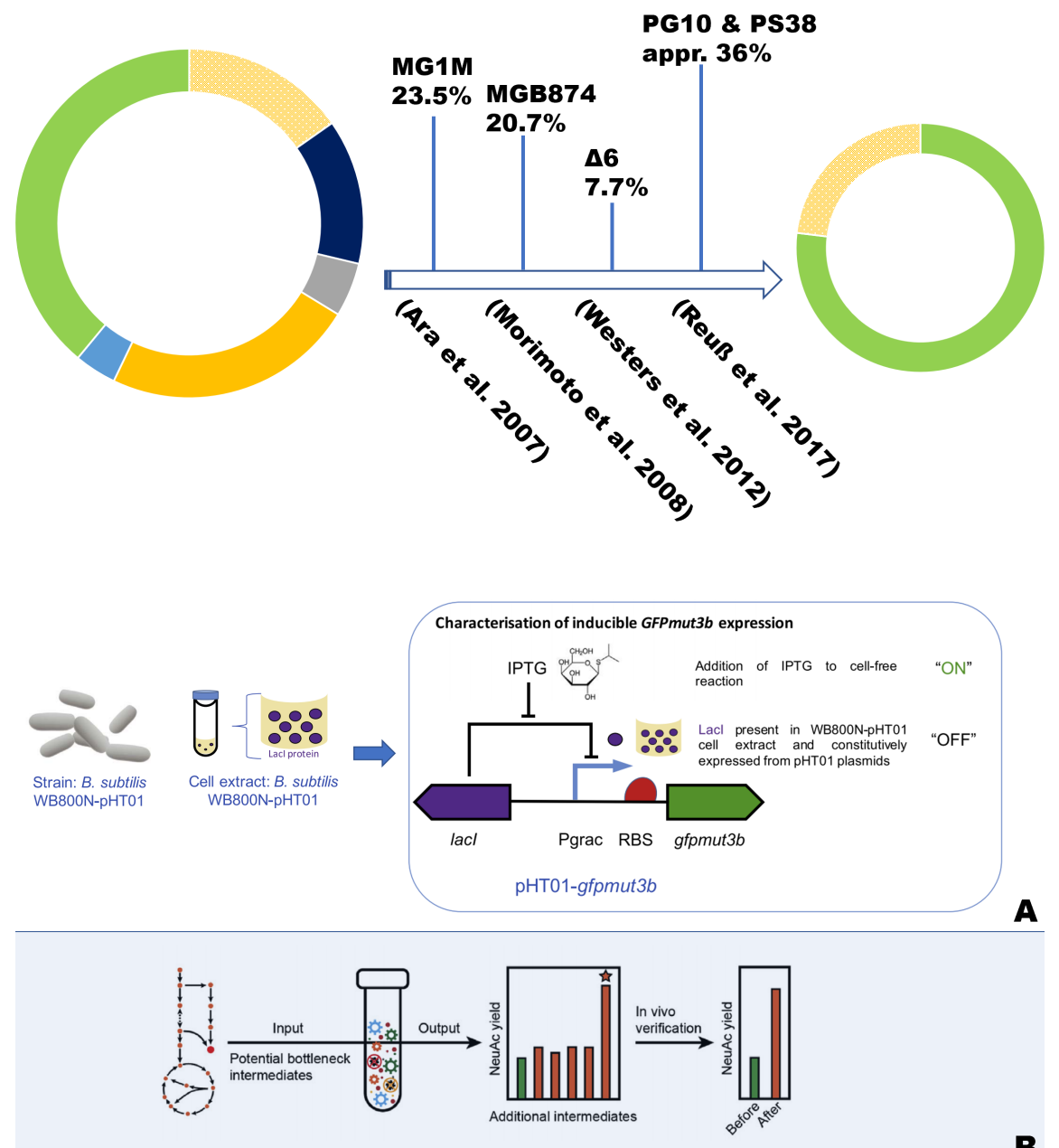

A

B 


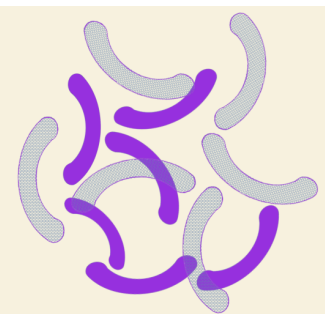

Monospecies

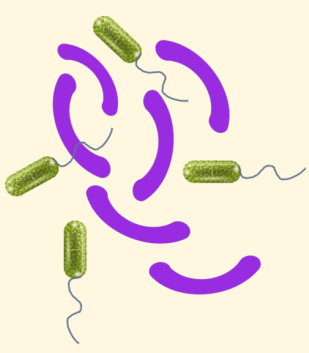

Multispecies

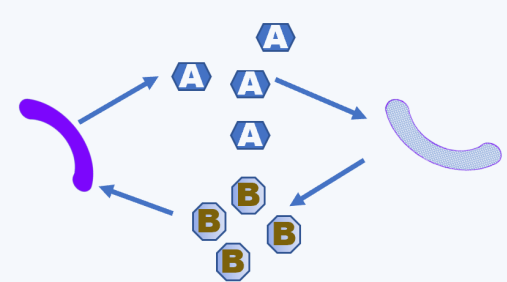

Metabolite exchange

C

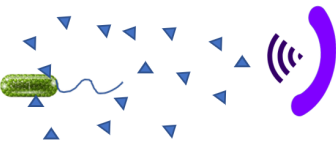

Signal transduction

D 